\title{
Host-Pathogen Molecular Factors Contribute to the Pathogenesis of Rhizopus spp. in Diabetes Mellitus
}

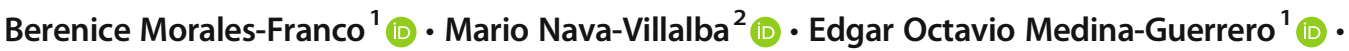 \\ Yaír Adonaí Sánchez-Nuño ${ }^{1}$ (D) Perla Davila-Villa ${ }^{2}$ (D) - Elsa Janneth Anaya-Ambriz ${ }^{1}$ (D) $\cdot$ Claudia Lisette Charles-Niño $^{1}$ (D)
}

Accepted: 20 November 2020

(C) The Author(s), under exclusive licence to Springer Nature Switzerland AG part of Springer Nature 2021

\begin{abstract}
Purpose of Review Infectious diseases represent up to $12 \%$ of all deaths in people with diabetes mellitus (DM). The development and progression of DM generate a chronic inflammatory state with unique characteristics that have been exploited by some pathogens; one of them is Rhizopus spp., a fungus considered the causative agent of mucormycosis. This disease has a poor prognosis with high mortality rates, and the apparition of resistant isolates each year has become a worrying concern. DM is an actual and continuing health problem, and for that reason, it is of foremost importance to study the pathogenesis of mucormycosis to generate new prevention and treatment strategies.

Recent Findings The worldwide incidence of mucormycosis has increased in recent years. The pathogenic mechanisms and factors identified in Rhizopus spp. are the cell wall, spore germination, proteins, and enzymes related to iron sequestration, $\operatorname{CotH}$ fungal protein, positive regulation of the GRP78 cell receptor, and immune evasion due to survival within phagocytes, among others. The physiopathology of DM offers favorable conditions for the successful replication of Rhizopus spp.

Summary The main reason for increase of incidence of mucormycosis caused by Rhizopus spp. has been associated with the rise of worldwide prevalence of DM. Knowing the fungal pathogenic mechanisms as well as the relationships between Rhizopus with the microenvironment found in the human body will undoubtedly help generate better antifungals to enhance treatment outcomes. Nowadays, some strategies to combat the fungus are based on the knowledge of its proteins, cellular interactions, and iron metabolism.
\end{abstract}

Keywords Rhizopus $\cdot$ Mucormycosis $\cdot$ Diabetes mellitus $\cdot$ Fungal infection $\cdot$ Evasion

\section{Introduction}

According to the World Health Organization, 422 million people worldwide live with diabetes mellitus (DM), 1.6 million die each year, and it has been estimated to be the seventh leading cause of death by 2030. Type 1 diabetes mellitus (T1DM) is an autoimmune disease and type 2 (T2DM) is

This article is part of the Topical Collection on Tropical Mycoses

Claudia Lisette Charles-Niño

hclcharles@gmail.com

1 Departamento de Microbiología y Patología. Centro Universitario de Ciencias de la Salud, Universidad de Guadalajara, Sierra Mojada 950, Edificio O, CP 44340 Guadalajara, Jalisco, Mexico

2 Laboratorio de Investigación en Patología. Centro Universitario de Ciencias de la Salud, Universidad de Guadalajara, Sierra Mojada 950, Edificio C, CP 44340 Guadalajara, Jalisco, Mexico related to environmental factors and represents almost 95\% of cases. The American Diabetes Association estimated the cost of diabetes in $\$ 327$ billion in 2017, the largest amount spent on treating comorbidities associated with a lack of glycemic control. Moreover, $12 \%$ of deaths in people with diabetes are attributed to infectious diseases [1-3]. A good example of DM vulnerability is the current global pandemic of SARS-CoV2, where many studies show DM as one of the most common comorbidities with an increased risk of disease complications $[4,5]$.

In general, DM is considered a risk factor for severe forms of soft tissue, urinary, and respiratory infections. The development and progression of DM generate a chronic inflammatory state that predisposes infection by Rhizopus spp., a fungus that is the causative agent of mucormycosis. The worldwide incidence of mucormycosis has increased in recent years, and some outbreaks of the disease have been reported recently [6-8]. The main reason for this increase has been associated 
with the rise of immunodeficiency conditions in the population. It is an opportunist infection and several risk factors associated with mucormycosis are male sex, age between 39 and 61 years old, having factors that trigger immune dysfunction like the use of steroids and immunosuppressive therapy, and infectious, metabolic, and hematological diseases [7-10]. Mucormycosis is also classified as a nosocomial infection since a small percentage is present in hospitalized patients due to the use of contaminated material, such as medical adhesive tapes or vascular devices. Likewise, studies have shown the existence of this pathology in patients without comorbidities and a good immune response $[8,11]$.

Mucormycosis has a poor prognosis with high mortality rates (70-95\%) primarily due to a late diagnosis from a deficient widespread availability of rapid and specific diagnostic assays (Soare AY, 2020). Treatment is aggressive and invasive because the removal of necrotic tissue is required to enhance the action of the antimycotic. Further concern involves the apparition of resistant isolates each year, representing an imminent public health problem [12]. These data sustain the fact that mucormycosis is a complex emergent disease that requires intensive studies. On the other hand, it should be noted that rhino-orbital-cerebral mucormycosis is the most frequent clinical presentation and the main risk factor is DM $[6,13]$. Worldwide more than $60 \%$ of patient with DM $(75 \%$ in México) have poor glycemic control, which makes them susceptible to opportunistic infections such as mucormycosis $[14,15]$. Considering that $\mathrm{DM}$ is an actual and continuing health problem, it is of foremost importance to study the pathogenesis of mucormycosis to generate new prevention and treatment strategies.

\section{Mucormycosis, a Challenging Emergence Disease}

Respiratory infectious diseases are predominant infections in DM population. Viruses and bacteria are the most frequent causal agents. It has been shown that 7 to $14 \%$ of COVID19 patients have DM as the main comorbidity $[16,17]$. There is a risk factor up to 3 times higher for developing tuberculosis or SARS, 5 to 6 times for severe infection by Influenzavirus, 7 times for severe disease by MERS-CoV, and 12 times for severe melioidosis infection when DM is present as a comorbid condition [4, 18-20]. However, fungal pathogens are also important causal agents because mycoses are more frequent, recurrent, and severe in patients with DM in comparison with non-DM patients.

Mucormycosis is an emergent and opportunist fungal disease that includes six main clinical presentations, rhino-orbital cerebral mucormycosis (ROCM), and pulmonary, cutaneous, gastrointestinal, renal, and disseminated mucormycosis. The prevalence of cases per million inhabitants of mucormycosis is 0.6 in Oceania, 1.8 in America, 1.8 in Africa, 8.3 in Europe, and 29.9 in Asia. In the United States of America (USA), the reported prevalence is 3.0 cases per million inhabitants. Nonetheless, due to the diagnostic complexity, a significant number of individuals are not diagnosed, and therefore, the numbers may be higher. The estimated cases in the world are 10,000 per year (Fig. 1) [6, 13]. In developing countries, the incidence of mucormycosis is linked to diabetes and emerging risk factors like chronic renal failure and hospitalization in intensive care units [6].

As an opportunistic disease, mucormycosis regularly occurs in immunosuppressed individuals. Regardless of the clinical forms of mucormycosis or its infection route (oral, respiratory or transcutaneous), fungal spores can frequently penetrate blood vessels and disseminate hematogenously and cause thrombosis and necrosis. The other route of transmission is by direct invasion of the cribriform lamina. Initial ROCM symptoms are similar to a sinus infection; the evolution of this pathology can lead to significant damage of the nervous system causing ocular alterations, such as blurred or double vision accompanied by pain. In addition, nerve injury may lead to paresthesia and other symptoms like fever and skin lesions within the nasal cavity. This fungus also causes a significant inflammatory response that could result in purulent arteritis, thrombosis, and tissue necrosis [21].

The immune system is capable of eliminating spores or hyphae easily by phagocytosis. In this context, any abnormalities in the immune system, especially in the innate response, constitute an opportunity for the growth of Rhizopus spp. and the development of mucormycosis. In fact, one of the first hematological findings in patients with suspicious lesions is neutropenia and, in this context, patients with neutropenia have nearly 2 times more risk of develop mucormycosis [22]. Other detection methods such as biopsies of lesions could be useful for the diagnosis of mucormycosis. The main biopsy findings include wide and coenocytic hyphae, inflammation, and polymorphonuclear infiltration, including plasma cells and eosinophils. Moreover, the presence of suppurative necrosis with infiltration of neutrophils, and giant or epithelioid cells is highly suggestive of mucormycosis [21]. On the other hand, some imaging techniques enable the diagnosis when the infection occurs through the direct invasion of the ethmoid sinuses. For instance, computed tomography (CT) and magnetic resonance imaging (MRI) have detected sinus opacification, bone erosion (osteomyelitis that may affect cranial nerves), and obliteration of deep fascia planes [21].

A timely diagnosis of mucormycosis is important for a good prognosis and disease resolution because treatment must focus on the patient's symptoms. Treatment usually consists of surgical treatments such as debridement accompanied by pharmacological treatment (amphotericin B) [21]. However, the mortality of mucormycosis remains high (approximately $70 \%$ ). Antimycotic treatment is complicated because the main 


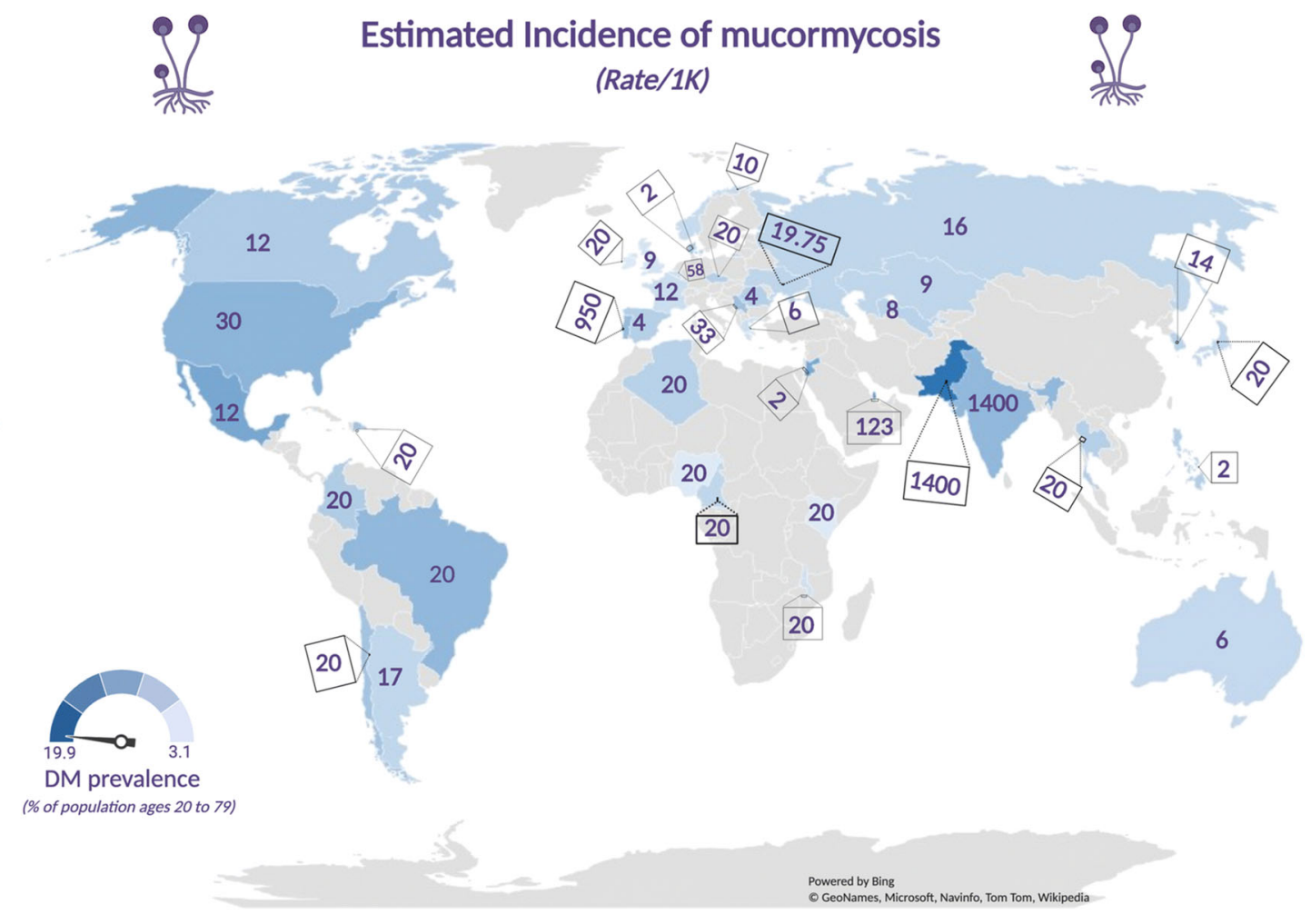

Fig. 1 Estimated prevalence of DM and incidence of mucormycosis. Data obtained from Prakash et al., 2019, and Atlas from International Diabetes Federation, 2019. It can be observed that in some countries with a high prevalence of DM they also present a high incidence of

underlying disease is diabetes mellitus often with kidney complications, and amphotericin B is nephrotoxic with a maximum tolerated dose up to $1 \mathrm{mg} / \mathrm{kg} / \mathrm{day}$. Mucorales species, including Rhizopus spp., are resistant to nystatin and triazoles; therefore, there are limitations in antifungal therapy [23]. In this regard, understanding the pathogenicity-associated factors that are involved in the development of mucormycosis is essential for the generation of new treatment strategies.

\section{Rhizopus spp. as an Etiological Agent of Mucormycosis}

Until now, species from more than 10 genera have been identified as causative agents of mucormycosis, where members of the Mucoraceae family are the most frequent (Rhizopus spp., Mucor spp., and Rhizomucor spp.) following the Lichteimiaceae family and the single member of the Cunninghamellaceae family (C. bertholletiae) [24]. In general, Rhizopus spp. is the most frequent genus related to mucormycosis and it is mainly associated with the most frequent clinical presentation, ROCM. In addition, it is one of the three agents with the highest mortality rates $(C$. bertholletiae $77 \%$, mucormycosis, such as Pakistan (19.9 and 1400 respectively), India (10.4 and 1400 respectively), Portugal (9.8 and 950 respectively), and Qatar (15.6 and 123 respectively) $[6,10]$

Rhizopus spp. 57\%, and Mucor spp. 41\%), primarily associated with diabetes as the comorbidity $[12,25,26]$.

The genus Rhizopus belongs to the Mucormycota division and the Mucorales order and is one of the 19-21 genera from the Mucoraceae family. The Mucorales order is characterized by non-septate mycelium, abundant anamorphic sporangiospores in a diverse-shaped columella within a sporangium and teleomorphic zygospores, often with specific ornamentation [27]. Rhizopus spp. is aerobic, thermotolerant, fast-growing, saprotrophic, and ubiquitous in soil, animal excrement, or bread and rotting vegetables. In addition, it has an optimal growth temperature of $39^{\circ} \mathrm{C}$ under conditions of low $\mathrm{pH}$ and high glucose concentration. On agar culture medium, Rhizopus spp. generates white cottony colonies that could change from gray or yellow and it also produces organic acids, ethanol, and hydrolytic enzymes [28].

Rhizopus genomes have an average size of $45 \mathrm{Mb}$, containing numerous simple sequence repeats, around $40 \%$ of GC content (guanine-cytosine content), which has a role in transposon biology and they are organized in 6 to 16 chromosomes $[29,30]$. There are 11 to 13 species described in the genus Rhizopus grouped in four complex species: $R$. microsporus, $R$. stolonifer, $R$. arrhizus (or $R$. oryzae), and $R$. delmar (R. arrhizus var. delemar). Compared with other species, 
R. arrhizus is most commonly isolated in clinical cases of mucormycosis with higher amylase and lipase activity and increased siderophore production.

\section{The Immunopathological Mechanisms of DM2 Enhance and Fuse with the Pathogenic Mechanisms of Rhizopus}

Despite the important role of Rhizopus spp. in agriculture, the food industry, biotechnology, and human medicine, the mechanisms involved in the growth, metabolism, and immune evasion have been understudied. The pathogenic mechanisms and factors identified in Rhizopus spp. include the cell wall, spore germination, proteins, and enzymes related to iron sequestration, CotH fungal protein, positive regulation of the GRP78 cell receptor, and immune evasion due to survival in phagocytes, among others. Most of them are successful due to immunosuppression or favorable conditions found in the host. The physiopathology of diabetes mellitus offers ideal conditions for the successful replication of Rhizopus spp., especially in those individuals with complications of uncontrolled hyperglycemia.

T2DM is a metabolic disease that causes persistent hyperglycemia and eventual insulin dysfunction. Hypertrophic obesity triggers the proinflammatory state broadly associated with the etiopathogenesis of T2DM. People with obesity and T2DM have an immunocompromised state characterized by dysregulated, dysfunctional, and/or unresponsive innate and adaptive immune cells $[19,31]$.

Some studies have shown dysfunctional components of the immune system due to a chronic inflammatory state. Patients with DM have low levels of the complement protein C4 associated with dysfunctional neutrophils and a decreased response to cytokines. Mononuclear cells and monocytes of DM release minor amounts of IL-1 and IL-6 in response to lipopolysaccharide (LPS), main component of the outer membrane of Gram-negative bacteria, that is activated in phagocytosis. Lymphocytes and macrophages have reduced IL-10 production due to increased glycosylation. Furthermore, it has been shown that patients with DM have a reduced major histocompatibility complex I (MHC-I) cell expression, which impairs cell-mediated immunity. Furthermore, DM reduces polymorphonuclear leukocyte mobilization, chemotaxis, and phagocytic activity [19, 31].

Phagocytic cells have an inflammatory role in poorly controlled DM since they cause the most frequent complications, such as infections or vascular damage. Mainly macrophages, but also monocytes, dendritic cells, neutrophils, and mast cells are phagocytic cells [32]. These cells by using metabolic pathways, such as glycolysis to produce energy in the form of ATP and pentose phosphate to generate free radicals, eliminate microorganisms that have the ability to invade the body (Fig. 2).
The main function of these cells is phagocytosis, but multiple functions including inflammation have undoubtedly been identified [33]. As the prevalence of DM2 increases, the number of infections caused by fungi such as Rhizopus spp. also increases. Recent experimental studies have associated this risk with the malfunction of phagocytic activity and the low percentage of cells responsible for this activity. Conditions such as hyperglycemia and acidosis could cause phagocytic cell dysfunction, thus increasing the risk of Rhizopus spp. infections.

Macrophages are phagocytes by excellence due to the fact that they have a broad collection of receptors, such as Toll-like receptors (TLR) or pattern recognition receptors (PRR) that allow the initiation of the phagocytosis process. There are two main subsets of macrophages, classically activated M1 macrophages and activated $\mathrm{M} 2$ macrophages. Classical activation requires stimuli as a trigger, such as IFN-g, TNF-a, LPS, or GM-CSF, and once activated, macrophages produce proinflammatory cytokines (IL-1 $\beta$, TNF, IL-12, and IL-18), synthesis of nitric oxide (NO), and the restriction of iron with an antimicrobial purpose. On the other hand, macrophages M2 are activated with the opposite pathway by CSF-1, IL-4, IL10 , IL-13, and TGF-b as they release IL-10 and low IL-12. In this context, M2 macrophages are called anti-inflammatory and they have been identified in allergic diseases, angiogenesis, and tissue remodeling and they are involved in the response against parasites and fungal cells [4, 34]. But, what happens in individuals with T2DM? (Fig. 2).

A well-documented and accepted hypothesis about the pathogenesis of T2DM suggests that high levels of glucose cause cellular hypoxia, endoplasmic reticulum (ER) stress, increased release of reactive oxygen species (ROS), free fatty acids (FFA), and cytokine production in the liver, muscle, and adipose tissue. Hypertrophic cells in adipose tissue release pro-inflammatory cytokines like interleukin-1 $\beta$ (IL$1 \beta$ ), tumor necrosis factor (TNF), and chemokines like CCchemokine ligand 2 (CCL2), CC-chemokine ligand 3 (CCL3), and CXC-chemokine ligand 8 (CXCL8). M1 macrophages are recruited by the action of TNF- $\alpha$, and its activation releases more pro-inflammatory cytokines (mainly IL-1 $\beta$ ) that generate persistent inflammation and the recruitment of more M1 macrophages (Fig. 2). In addition, at the cytoplasm level of tissue cells, FFA are recognized by TLR4 activating JNK-AP-I and IKK-NFKB signaling [18, 19, 31]. By doing so, the expression and release of proinflammatory cytokines are increased promoting the local inflammatory state. Infiltration of diabetic tissue with M1 macrophages could contribute to the resistance of Rhizopus spp. to phagocytosis since M2 macrophages appear to be better able to activate and then kill fungal cells. For this reason, it is convenient to carry out specific studies that demonstrate the causes of these defects to further develop new therapeutic or preventive measures [35]. 


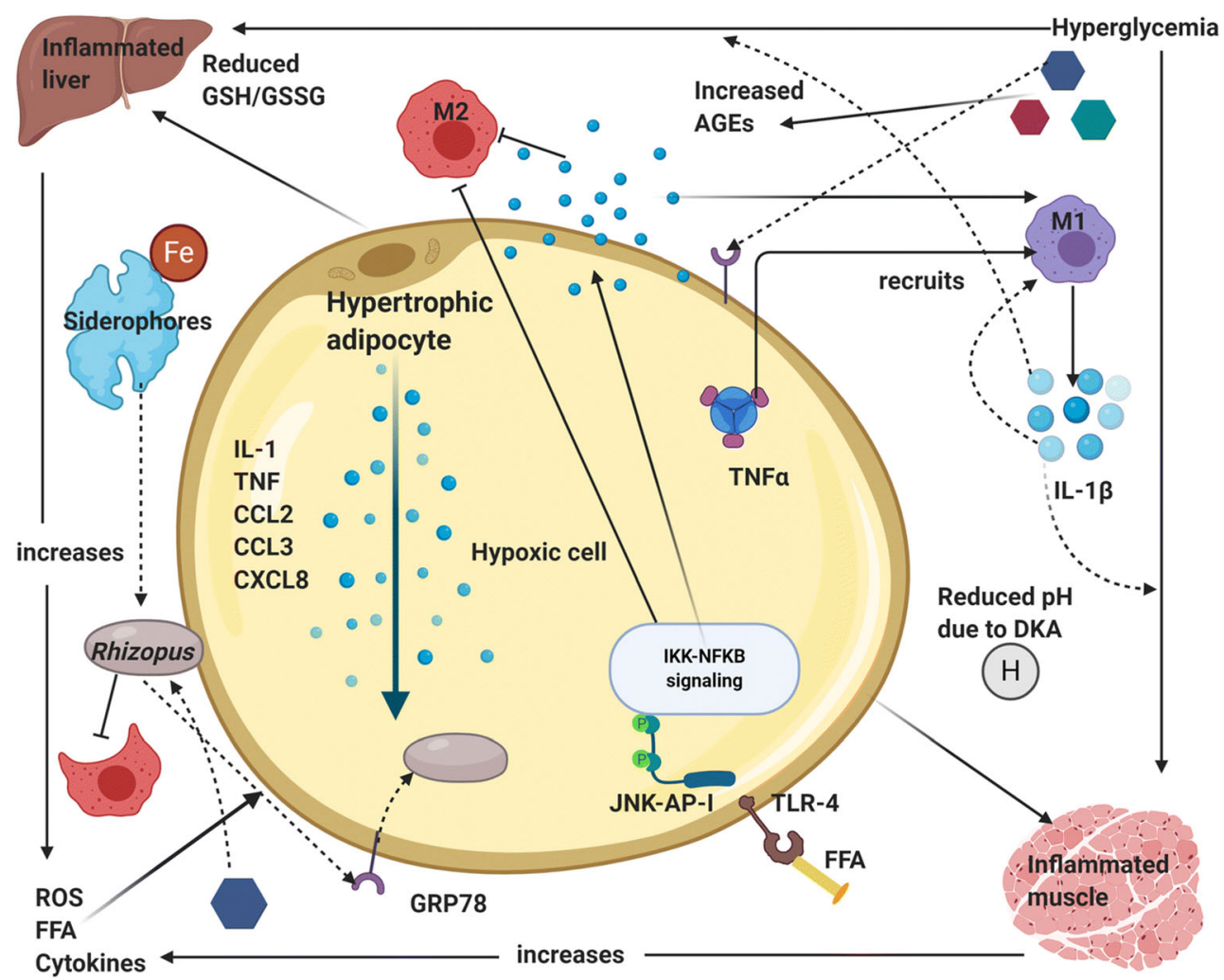

Fig. 2 Immunopathogenesis of comorbidity DM-mucormycosis. High levels of glucose cause endoplasmic reticulum (ER) stress and increased release of reactive oxygen species (ROS), free fatty acids (FFA), and cytokines in the liver, muscle, and adipose tissue. Hypertrophic cells in adipose tissue release pro-inflammatory cytokines like interleukin- $1 \beta$, tumor necrosis factor alpha $(\mathrm{TNF}-\alpha)$ and chemokines like CC-chemokine ligand 2 (CCL2), CC-chemokine ligand 3 (CCL3), and CXC-chemokine ligand 8 (CXCL8). M1 macrophages are recruited by the action of TNF- $\alpha$ and its activation releases more pro-inflammatory cytokines (mainly IL-1 $\beta$ ) that generate persistent inflammation and the recruitment of more M1 macrophages. In addition, at the cytoplasm level of tissue cells, FFA are recognized by Toll-like receptors 4 (TLR-4) activating JNK-AP-I and IKK-NFKB signaling. Afterwards, the expression and release of proinflammatory cytokines promote the local inflammatory state. There is a tissue infiltration of M1 macrophages in

Some studies have found that the immune system elicited by supplementing high-quality proteins in the diet known as nutritional immunity and triggered by iron restriction inside macrophages inhibits Rhizopus growth by a correct phagosome maturation. Patients with mucormycosis show an intracellular persistence in alveolar macrophages (AM), where it was found that Rhizopus conidia had melanin surface retention in order to arrest the maturation of LC3-associated phagosome, which also impact IFN- $\gamma$ secretion. Iron uptake-related mechanisms are a promise for future therapeutics against the infection, since apoptosis is also triggered in Rhizopus when there are irondeprived conditions [36].

The human body needs iron for many vital processes, such as cell growth and development, but this element needs to be bound to host carrier proteins (transferrin, ferritin, and lactoferrin) to avoid the toxic effect of free iron. On the other diabetic patients, promoting an M1 macrophage pro-inflammatory response instead of an M2 macrophage regulatory response. Rhizopus uses iron (throughout siderophores) and glucose (which serum levels are increased in type 2 diabetes mellitus subjects due to the diminished cell catchment for insulin resistance) as growth factors and energy source, respectively. Low $\mathrm{pH}$ in patients with diabetic ketoacidosis (DKA) generates more susceptibility to mucormycosis because of the generalized oxidative environment which affects glutathione renovation through GSH/GSSG enzyme cycle. Rhizopus inhibits phagocytosis or survives to it. Advanced glycation end products (AGEs) and reactive oxygen species derived from the increased glucose metabolism accumulate in organs and tissues triggering the typical micro- and macrovascular alterations in DM patients leading to an increased susceptibility to a Rhizopus infection

hand, Mucorales use free iron for their own processes; therefore, they must obtain it through multiple mechanisms to convert it into free iron from sources available in the host, or by taking advantage of the host's altered defenses, when there is an increased amount of free iron [37,38]. In this context, patients with diabetic ketoacidosis (DKA) requiring hemodialysis with deferoxamine chelation treatment have augmented levels of serum free iron, and for that reason, they are more likely to develop mucormycosis $[39,40]$.

Several studies show that iron has an important role in Rhizopus, and like in other fungi, iron is obtained from the host by two mechanisms, either using siderophores (iron chelators) or high-affinity iron permeases [40-43]. Studies in Rhizopus have identified key molecular determinants of iron assimilation, one of them is called high-affinity system, which is composed of ferric reductase (Fre), ferroxidase (Fet3), and 
high-affinity iron permease (FTR1) [44]. The high-affinity system reduces the free ferric ion $\mathrm{Fe}^{3+}$ to $\mathrm{Fe}^{2+}$ by ferric reductase (encoded by fre genes) to obtain a more soluble $\mathrm{Fe}$ form, then $\mathrm{Fe}^{2+}$ is oxidized by ferroxidase (encoded by $f e t 3$ genes) to obtain again $\mathrm{Fe}^{3+}$, which is recognized by the high-affinity iron permease (encoded by ftr 1 genes) that in the end concede the transport inside the cell $[42,45]$. The high affinity system is also regulated by the environmental levels of iron and it is activated when there is low availability of it, which allows Rhizopus to obtain the highest amount of iron present in the environment. This system is considered a crucial virulence factor since the depletion of the high-affinity iron absorption system has been shown to reduce virulence, trigger growth defects, and upon iron starvation, even induce apoptosis in the fungus [46].

In the siderophore system, fungi compete with the host for the available iron. Fungi siderophore may be intrinsic or extrinsic and Rhizopus uses both. Rhizoferrin is the main intrinsic siderophore synthesized by Rhizopus, and it takes iron through a receptor-mediated and energy-dependent process. A genome-sequencing study of $R$. oryzae demonstrates 13 possible siderophore permeases that might act as receptors for siderophores. Through several protein crystallography assays, it has been shown that rhizoferrin has a diaminobutane backbone attached to two citric acid residues with an R, Rconfiguration around a chiral center. However, specific mechanisms of virulence have not yet described for rhizoferrin in contrast with a xenosiderophore desferrioxamine, which chelates iron from transferrin and transports it inside by the highaffinity iron complex [46].

In patients with diabetes, the fasting state generated by the lack of sufficient levels of insulin triggers the activation of the metabolism of amino acids and triacylglycerol (TAGs) stored in adipose tissue as an energy source. Serum concentrations of glycerol and free fatty acids are elevated due to restricted lipolysis, as well as alanine due to muscle catabolism. Glycerol and alanine are substrates for hepatic gluconeogenesis stimulated by excess glucagon that accompanies insulin deficiency. Glucagon also stimulates the mitochondrial conversion of free fatty acids to ketones. Under normal conditions, insulin blocks ketogenesis by inhibiting the transport of free fatty acid derivatives to the mitochondrial matrix, but in the absence of insulin, ketogenesis proceeds [19]. As a product of TAG metabolism, abundant ketone bodies are generated and thus influence serum $\mathrm{pH}$, and this leads to the dysfunction of many serum enzymes. Some of them are transferrin and hemoglobin, which at a $\mathrm{pH}$ of 6.88-7.3 remain protonated and incapable of transporting $\mathrm{Fe}+3$, so in patients with $\mathrm{DM}$ there is a greater amount of $\mathrm{Fe}+3$ available in serum. In addition to use the available $\mathrm{Fe}+3$ in these patients, Rhizopus have a ketone reductase enzyme that allows the development of the fungus in this acidic state [45, 47]. Other host enzymes are affected by the acidosis generated by Rhizopus spp. and this has a direct impact on phagocytosis and chemotaxis. Furthermore, it has been reported recently that reduced iron levels trigger the M1 proinflammatory LPS-induced response; thus, another mechanism adds to the polarization of an unfavorable response to fungal clearance [48].

It has been reported that ketoacidosis alone does not predispose to mucormycosis by Lichtheimia; however, DKA causes overexpression of Mucorales proteins and host surface receptors that augment the binding to endothelium and thus increase the risk of fungal invasion [49]. Main alterations in host enzymes caused by ketoacidosis are reversible after improvement in environmental $\mathrm{pH}$; however, if hyperglycemia persists, irreversible dysfunction of many enzymes is possible [50]. For this reason, early detection of ketoacidosis is important in patients with DM. Timely treatment focused on reversal of the acidosis state could contribute to reduce the patient's susceptibility to Mucorales invasion and other infections.

There is another factor to consider for a good phagocytic response: reactive oxygen species (ROS). Oxidative stress is important in other virulence factors that will be discussed later. In individuals with diabetes, as a consequence of insulin resistance, hyperglycemia continues, and in order to reduce the glucose levels, there is an increased secondary lipolysis and glucose metabolism through oxidative phosphorylation. Advanced glycation end products (AGEs) and reactive oxygen species derived from the increased glucose metabolism accumulate in organs and tissues triggering the typical micro and macrovascular alterations in DM patients [51]. Oxidative stress is poorly controlled in these patients because the main antioxidant system of glutathione (GSH/GSSG) is affected by the deficiency of the cofactor NADPH needed for the regeneration of reduced glutathione. NADPH deficiency is due to its fast consumption by the polyol pathway for glucose metabolism. Oxidative stress activates inflammation mediated by NF-kB and TLR receptors; hence, a persistent chronic inflammatory state is eventually generated [52].

\section{Overexpression of GRP78 Protein Caused by DM2 Is Suitable for Host Invasion by Rhizopus}

Glucose-regulated proteins (GRPs) were first observed in transformed fibroblasts, in which the synthesis of these proteins increased when glucose depletion was induced [53]. The most abundant GRP is a $78-\mathrm{kDa}$ glucose-regulated protein (GRP78), also known as immunoglobulin-binding protein (BiP), located in the lumen of the endoplasmic reticulum and expressed in mammalian cells. GRP78 is encoded by the HSPA5 gene, assigned to chromosome $9 \mathrm{q} 34$. Structurally, GRP78 consists of two functional domains: a nucleotide-binding domain (NBD) and a substrate-binding domain (SBD), and its activity is regulated by the allosteric 
ATPase cycle, where NBD binds and hydrolyzes ATP, and SBD binds to polypeptides [54-56]. While new features have come to light in recent years, GRP78 has been traditionally considered a molecular chaperone belonging to the HSP70 family, which acts to control ER stress through regulation of the unfolded protein response (UPR), and it plays a key role in the folding, assembly, and quality control of proteins and misfolded protein degradation $[57,58]$.

As mentioned earlier, GRP78 is mainly found in the ER, but it also has the ability to translocate and accumulate in other intracellular locations [59]. In the cytosol, GRP78 protein is found as GRP78va, an isoform generated by alternative splicing (retention of intron 1) and alternative translation, which lacks the ER signal peptide. GRP78va has cytoprotective properties and the potential to regulate signaling to the UPR, promoting cell survival under stress from the cytosol [60]. The expression of GRP78 in the intermembrane space, internal membrane, and the matrix of mitochondria is triggered by ER stress, and it participates in UPR signaling inside the organelle [61]. The recent relevance of GRP78 expression is its translocation to the surface of the cell membrane, where it has receptor and regulatory functions in cell signaling by the formation of complexes with extracellular ligands and proteins anchored to the cell surface [59, 62]. In 1997, the GRP78 protein was identified in the cell surface of malignant lymphocytes in patients with acquired immunodeficiency syndrome (AIDS) and cutaneous lymphoma or leukemia [63]. Since then, this protein has been constantly analyzed and is now known to be involved in the proliferation of diverse types of cancer, chronic or inflammatory diseases, as well as the invasion by fungi and some viruses [59]. Thus, the stress in the ER, caused by many pathologies, is known to trigger the GRP78 translocation to the cell surface, where it is called csGRP78, and it can even have antigenic properties inducing the production of anti-GRP78 autoantibodies and it also acts as an associated signal receptor to the membrane [64].

Recent studies have shown that hyperglycemia is a stress trigger in the endoplasmic reticulum, which consequently induces the overexpression of the GRP78 protein depending on glucose concentrations and the persistence of high glucose levels. It has been proposed that GRP78 is translocated to the cell surface from the ER through a mechanism regulated by the MTJ-1 chaperone. Once this protein is located on the cell surface, it interacts with the $\beta 1$ integrin in charge of mediating phosphorylation of kinases. The activation of the Kinase of Focal Adhesion (FAK) and downstream protein kinase Akt causes fibrosis through the expression of extracellular matrix proteins $(\mathrm{ECM})$, such as fibronectin and type I collagen [65]. On the other hand, studies have shown that the overexpression of csGRP78 plays an important role as the entry receptor of some pathogens, like the Dengue virus, Ebola virus, Coxsackievirus, and the novel SARS-CoV2, among other viruses and Rhizopus spp. [66-68]. A mouse mucormycosis model has shown that high glucose levels increase 2- to 5-fold higher levels of Grp 78 mRNA in the sinus, lungs, and the brain compared with normal mice and it increased endocytosis up to $40 \%$ of $R$. oryzae by human endothelial cells [66]. These first assays suggested that csGRP78 is crucial for invasion but not for adhesion because it is bound by germlings but not by spores. However, it is highly probable that local factors, such as stasis and dehydration of the mucous secretion, superficial lacerations, or retentive anatomical niches, allow the establishment of sporangiospores and their subsequent germination into hyphae.

In addition, it was shown that Rhizopus delemar interacts with different receptors in nasal and alveolar epithelial cells. csGRP78 is overexpressed in nasal epithelial cells but not in alveolar epithelial cells when they are infected with Rhizopus spp. in vitro. Moreover, it was identified that Rhizopus spp. interacts with alveolar epithelial cells by bounding with integrin- $\beta 1$ and not with csGRP78. Therefore, microenvironmental conditions drive the pathogenicity of Rhizopus spp. where glucose, iron, and diabetic ketoacidosis (DKA) trigger csGRP78 overexpression only in nasal epithelial cells, but they do not trigger integrin- $\beta 1$ overexpression in alveolar epithelial cells. It is possible that this phenomenon is the explanation of the major clinical manifestation of mucormycosis in DM patients known as the rinocerebral presentation, in contrast with other immunodeficiencies where the pulmonary presentation is most frequent [69]. The diversity on cell receptor usage by Rhizopus spp. is mediated by the second component in this interaction; csGRP78 specifically interacts with homologous spore coating proteins $(\mathrm{CotH})$ present in the Mucorales, allowing invasion and damage to endothelial cells $[66,67,69,70]$.

$\mathrm{CotH}$ is a protein kinase belonging to the spore coating protein family, localized and required for the assembly of proteins in the inner layer of the spore coat; it is expressed during sporulation; and its activity is regulated by autophosphorylation with ATP and subsequent phosphorylation of $\mathrm{CotB}$ and $\operatorname{Cot} \mathrm{G}$ on serine residues. CotH has a short half-life of 4 to $5 \mathrm{~h}$ and its concentration falls shortly after the transcription of the structural gene has been deactivated. This protein has been recently identified and is a required component for spore germination in many bacterial and eukaryotic species, including human pathogens, such as spore-forming bacteria Bacillus anthracis and spore-forming fungi Rhizopus oryzae $[67,71,72]$. When GRP78 was identified as the necessary receptor for Mucorales invasion [66], the search for the putative ligand allowed the identification of $\mathrm{CotH}$ in Mucorales [67]. Thus, it has been possible to identify the expression of the $\mathrm{CotH1}, \mathrm{CotH} 2$, and $\mathrm{Cot} H 3$ genes in a variety of members of the Mucorales order. However, in the DKA context, evidence has been shown that $\mathrm{CotH} 3$ is mainly expressed in the germinations of $R$. oryzae and shows a greater capacity to adhere and, therefore, invade endothelial and nasal epithelial cells. Some studies have established that when 
exposing endothelial cells to germination of $R$. oryzae, the proteins that specifically reflect a greater expression on these include $\mathrm{CotH} 2$ to a lesser extent and $\mathrm{CotH} 3$ with a relatively higher proportion, which were recognized as the specific fungal ligands that bind to the GRP78 protein, promoting the invasion of the host endothelial/nasal epithelial cells [67, 69, 70, 73]. (Fig. 3). In contrast, in pulmonary mucormycosis, $\mathrm{CotH} 7$ is the major ligand that interacts with integrin- $\beta 1$ of alveolar epithelial cells and it is not closely related with $\mathrm{CotH} 3$ ( $\sim 50 \%$ amino acid identity) [69].

The mechanisms through which the interaction between the invading fungi and the endothelial/epithelial cells are promoted begin to have a solid foundation and represent an important step in the pathogenesis of diabetes-associated mucormycosis $[66,67,69]$. High glucose, iron, and Bhydroxy butyrate (BHB) as the predominant ketone body improve the growth of the fungus through inducing the expression of CotH3 in the DKA context [74]. The surface relocation of the GRP78 protein that copes with the stress of the endoplasmic reticulum caused by hyperglycemia and an acid microenvironment promotes a tissue stage predisposed for the establishment of Rhizophus spp. In this same tissular niche, the iron release from sequestered protein transferrin occurs through glycosylation or protonation processes. Therefore, high concentrations of glucose, the available free iron [36], and an acid microenvironment enhance $\mathrm{CotH}$ expression on the fungal cell surface, resulting in GRP78/CotH3 interaction and allowing endothelial/epithelial invasion and fungal dissemination [74]. After invading the superficial nasal epithelium, the fungus must interact with its basement membrane, since spores and stem cells from germ tubes bind to components of the extracellular matrix. This is supported through the observation of Rhizopus spp. attaching to plates coated with laminin and collagen IV [75].

In neutropenic mice infected with $R$. delemar strains, polyclonal antibodies of the peptide regions of CotH3 (antiCotH3) showed reduction in their fungal load, by preventing invasion and decreasing their subsequent hematogenous

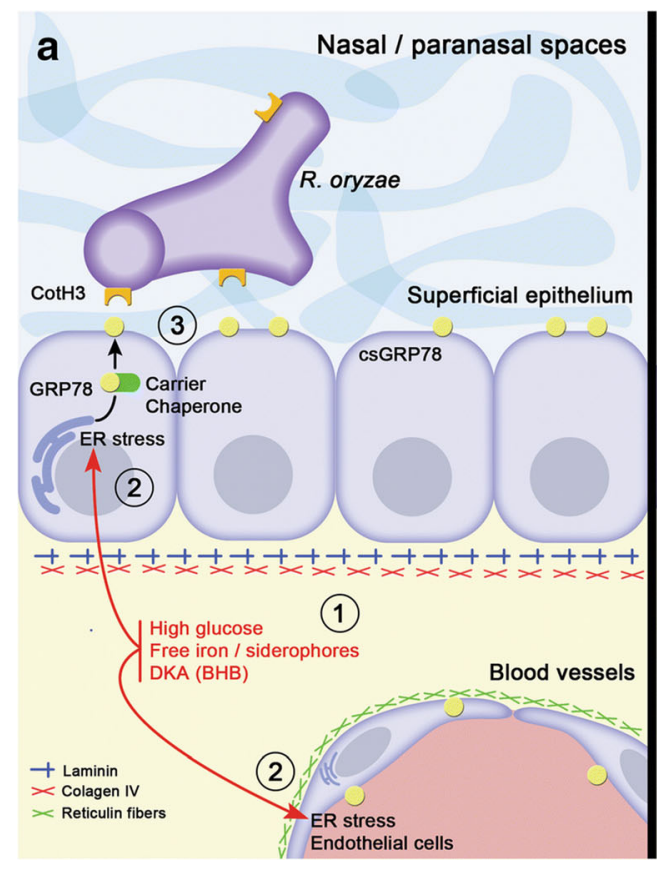

Fig. 3 Proposed mechanisms for the establishment of rhino-orbitalcerebral mucormycosis in diabetic patients. a (1) Tissular microenvironment in a diabetic patient is altered; there are high levels of glucose, free iron, and ketone bodies (BHB, B-hydroxy butyrate); and these conditions cause stress on the endoplasmic reticulum in the adjacent epithelial/endothelial cells. (2) In response to ER stress, GRP78 is overexpressed and relocated in diverse cellular compartments, particularly on the cell surface, carried by several co-chaperone proteins like MTJ-1 and Par-4. (3) Once GRP78 is exposed on the cell surface (csGRP78), it favors the possibility of interaction with the hyphae of $R$. oryzae through the expression of their CotH3 proteins. b (4) The interaction between the GRP78 and CotH3 proteins promote hyphae that can damage cells and penetrate the epithelium. (5) The association of $R$. oryzae hyphae to laminin and type IV collagen in the basement membrane allows their adherence and entry into the interstitium where the fungi can approach blood vessels. The microenvironmental imbalance

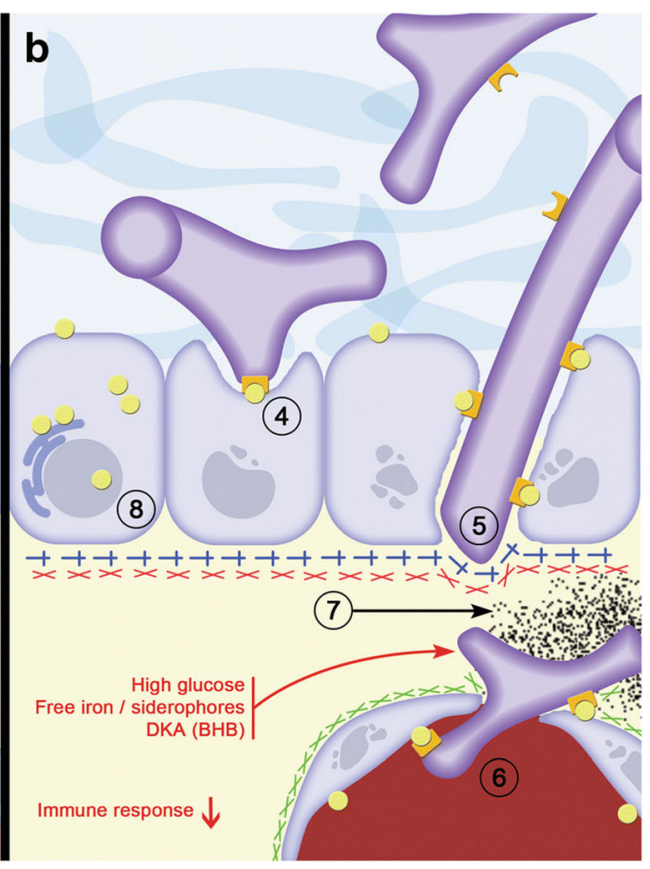

offers favorable conditions for fungus growth: energy (high glucose), free iron for its metabolic requirements, as well as a conducive acidic environment generated by ketone bodies. (6) Meanwhile, endothelial cells continue to produce GRP78 in all compartments and the hypha can associate with these proteins on the basal side where the presence of reticulin fibers are exceeded, which allows it to anchor and outflank this area to later interact with GRP78 expressed on the luminal surface of endothelial cells. Once internalized in the lumen of the blood vessels, the fungi consequently induce the extrinsic coagulation pathway activation, consequently cell damage occurs, and all this triggers the formation of thrombus. (7) This results in ischemia and sustained hypoxia, which generates infarction of the tissues and its consequent necrosis. (8) Finally, the altered microenvironment in diverse tissular compartments of these patients generates GRP78 overexpression, allowing Rhizopus spp. to find this protein in any type of epithelial or endothelial cell, establishing its interaction and continuing its invasive behavior 
spread and recruiting phagocytes and the elimination of fungi through opsonophagocytosis. Unfortunately, the presence of natural anti-CotH3 antibodies generated in exposed volunteers and patients infected with mucormycosis are too low to exert a protective activity as the one provided by anti-CotH3 in a murine model [67]. Nonetheless, other cellular and fungal proteins may be involved in mucormycosis pathogenesis because in vitro assays with different epithelial cells by using anti-CotH antibodies did not completely block $R$. delemarmediated damage of host cells [69]. However, improving the generation and use of anti-CotH3 antibodies represents a promising immunotherapeutic alternative.

\section{Rhizopus spp. Interactions: New Approaches for Treatment}

A recent study found that it is possible that bacterial endosymbionts of Rhizopus spp. have an effect on the virulence by innate immune evasion. The bacterium Ralstonia pickettii in endosymbiosis with Rhizopus microspores increases its ability to survive within macrophages, which attack and trigger a profuse pro-inflammatory cytokine release. $R$. pickettii is a Gram-negative bacillus that is considered a nosocomial pathogen frequently found contaminating medical solutions as sterile water, disinfectants, and saline solution. It is possible that $R$. pickettii participated in the last mucormycosis outbreaks reported in hospitals [8].

Patients with diabetes and allogeneic hematopoietic stem cell transplantation (another common mucormycosis risk group) have recently shown oral dysbiosis characterized by an increment of $R$. pickettii proportions [76]. This finding could have an impact in the incidence of nosocomial mucormycosis. An increased presence of $R$. pickettii in patients with diabetes and obesity has been reported. Therefore, Rhizopus infection in these patients could be due to the possibility that both microorganisms coincide and benefit each other, precisely in this type of patients. New approaches based in re-establish microbiota in DM patient could be useful.

Amphotericin B, posaconazole, and isavuconazole are the best available antifungals for the treatment of mucormycosis. A recent analysis of matching cases showed that new formulations of posaconazole were associated with lower mortality rates and favorable responses of up to $80 \%$ in invasive mucormycosis compared to amphotericin B [77]. Voriconazole, a polyene derived from triazole and better tolerance than amphotericin B showed that in 25 Mucorales strains (of which 18 were of the genus Rhizopus), voriconazole/amphotericin B combinations were more effective at inhibiting the growth of Rhizopus spp. in vitro and using the Galleria mellonella mucormycosis model compared to amphotericin B [78]. However, the results of the few analyzes performed in recent years with small size samples should be considered with caution. Therefore, the study of competitive interactions between Rhizopus and other microorganisms have been of interest for the search of new antifungals.

Evidence of competitive interactions between fungi and bacteria has been used for the development of antifungals. A recent study described use of tanzawaic acids from hot spring-derived Penicillum sp. as a lethal dose-dependent agent against Rhizopus oryzae, with similar efficacy to that obtained with amphotericin B and with no effect against other fungi such as Candida spp., Aspergillus spp. or Cryptoccoccus spp. [79]. Other study has shown that bacterial strains such as Serratia marcenses, Serratia proteamaculans, Bacillus subtillis, and Neurospora crassa significantly inhibit the growth of Mucor circinelloides, Actinomucos elegans, and Rhizopus stolonifer within 24$72 \mathrm{~h}$ after interaction with these bacterial strains or with derived volatile compounds [80].

On the other hand, the fungus cell wall is a mechanism of pathogenicity with the ability to evade interactions, mainly with immune system cells. There is a replication of some gene families in the genome of Mucormycotina subdivision lineage that suggests a whole genome duplication event as an evolution response for adaptation to the environment [29]. Chitin deacetylase and chitin deacetylases families of proteins are widely and diversely represented in the Rhizopus oryzae genome. These two enzymes are involved in chitosan or chitin synthesis, which is the main component of the fungal cell wall, and thus are involved in evading the immune system [81]. However, there are still no antifungal against this potential target.

Spore germination is the crucial mechanism associated with the pathogenic mechanism of angioinvasion, thrombosis, and tissue necrosis; some triggers of this mechanism are changes of nutrients, light, osmolarity, and $\mathrm{pH}$ among others. Experiments using $R$. delemar have shown that ungerminated spores have more than 6000 significantly expressed genes from germinated ones. Within 6 to $12 \mathrm{~h}$ of hyphal growth, the most relevant genes with augmented expression were those involved in mitochondrial enzymes, organophosphate and sulfur metabolism, transposase, ATPase, nucleoside triphosphatase, and a vast repertory of stress response genes [24]. Phase differences in proteome make difficult to generate vaccines or identify important antigenic compounds.

$R$. oryzae is a complex fungus to be manipulated in the laboratory and presents many challenges for the in vitro experimentation, thus limiting the research about virulence mechanisms. The inefficiency of null mutant retrieval because $R$. oryzae appears to have multiple copies of its genes has recently been resolved using state-of-the-art techniques such as genome editing using clustered regularly interspaced short palindromic repeats (CRISPR)/CRISPR-associated protein 9 
(Cas9), where by this technique a point mutation was induced in $R$. oryzae [82]. Although the RNA interference and CRISPR/Cas9 approach have been more used in other fungi, this first relatively easy manipulation performed in Rhizopus will undoubtedly bring new findings about virulence mechanisms in the following years.

\section{Conclusion}

Fungal pathogens such as Rhizopus spp. have been little studied. The uncontrolled increase in metabolic diseases in recent years has generated favorable microenvironments for the development of mucormycosis; Rhizopus spp. is a fungus whose genome contains information for its adaptation to different environmental conditions, so the human body offers a very favorable microenvironment in patients with immunocompromised conditions and chronic inflammation, such as those caused by diabetes mellitus. Although the treatment of mucormycosis is based on the control of DM characterized by immunosuppression, there is a critical need to identify new prophylactic measures or therapeutic targets against Rhizopus spp.

However, progress in this area has been very limited in recent years. There are still no approved vaccines against fungal pathogens and the availability of drugs for the treatment of mucormycosis is limited to a few. Although promising treatment alternatives such as the use of in vitro-reactivated Tspecific lymphocytes or the use of heat-killed yeast, Saccharomyces cerevisiae, as a candidate vaccine, have recently been reported, there have been no other approaches in recent years. It is necessary to continue deepening knowledge about the pathogenic mechanisms, the relationships between Rhizopus, and the microenvironment found in the human body. This knowledge, as well as the use of cutting-edge techniques such as CRISPR/Cas9 for the genetic modification of the complex genome of $R$. oryzae, will undoubtedly contribute to develop better antifungal agents, treatment alternatives, and vaccine development.

Acknowledgments We appreciate the training support for AnayaAmbriz EJ and Sánchez-Nuño YA, received from the Maestría en Microbiología Médica at UDG and the scholarship that they receive from CONACYT. Also, the training support for Morales-Franco B received from Doctorado en Farmacología at UDG.

\section{Compliance with Ethical Standards}

Conflict of Interest The authors declare that they have no conflict of interest.

Human and Animal Rights and Informed Consent This article does not contain any studies with human or animal subjects performed by any of the authors.

\section{References}

Papers of particular interest, published recently, have been highlighted as:

- Of importance

- Of major importance

1. Zhou C, Byard RW. An analysis of the morbidity and mortality of diabetes mellitus in a forensic context. J Forensic Sci. 2018;63: $1149-54$.

2. Centers for Disease Control and Prevention. National Diabetes Statistics Report, 2020. Atlanta, GA: Centers for Disease Control and Prevention, U.S. Dept of Health and Human Services; 2020.

3. Yang W, Dall TM, Beronjia K, et al. Economic costs of diabetes in the U.S. in 2017. Diabetes Care. 2018;41:917-28.

4. Mozafari N, Azadi S, Mehdi-Alamdarlou S, Ashrafi H, Azadi A. Inflammation: a bridge between diabetes and COVID-19, and possible management with sitagliptin. Med Hypotheses. 2020;143: 110111.

5. Tadic M, Cuspidi C, Sala C. COVID-19 and diabetes: is there enough evidence? J Clin Hypertens. 2020;22:943-8.

6.• Prakash H, Chakrabarti A. Global epidemiology of mucormycosis. J Fungi (Basel). 2019;5. https://doi.org/10.3390/jof5010026. This article shows and explains the increased incidence and prevalence of mucormycosis in the globe, mainly in T2DM subjects, thus showing the need of more studies and research on this field of knowledge.

7. Walther, G., Wagner, L. \& Kurzai, O. Outbreaks of Mucorales and the Species Involved. Mycopathologia. 2020; 185, 765-781. https://doi.org/10.1007/s11046-019-00403-1.

8. Nasir N, Sayeed MA, Jamil B. Ralstonia pickettii Bacteremia: an emerging infection in a tertiary care hospital setting. Cureus. 2019;11. https://doi.org/10.7759/cureus.5084.

9. Castillo Garcia LA. Experiencia de mucormicosis en el Hospital Juárez de México. Rev Hosp Jua Mex 2004; 71(1):3-13.

10. IDF Atlas 9th edition and other resources, https://www. diabetesatlas.org/en/resources/. Accessed 21 September 2020.

11. Castrejón-Pérez AD, Miranda I, Welsh O, et al. Cutaneous mucormycosis. An Bras Dermatol. 2017;92:304-11.

12. Jeong W, Keighley C, Wolfe R, Lee WL, Slavin MA, Kong DCM, et al. The epidemiology and clinical manifestations of mucormycosis: a systematic review and meta-analysis of case reports. Clin Microbiol Infect. 2019;25:26-34.

13. Patel A, Kaur H, Xess I, et al. A multicentre observational study on the epidemiology, risk factors, management and outcomes of mucormycosis in India. Clin Microbiol Infect. 2020;26:944.e9944.e15.

14. Chan JCN, Gagliardino JJ, Baik SH, Chantelot JM, Ferreira SRG, Hancu N, et al. Multifaceted determinants for achieving glycemic control the international diabetes management practice study (IDMPS). Diabetes Care. 2009;32:227-33.

15. Corzo-León DE, Chora-Hernández LD, Rodríguez-Zulueta AP, Walsh TJ. Diabetes mellitus as the major risk factor for mucormycosis in Mexico: epidemiology, diagnosis, and outcomes of reported cases. Med Mycol. 2018;56:29-43.

16. Li B, Yang J, Zhao F, Zhi L, Wang X, Liu L, et al. Prevalence and impact of cardiovascular metabolic diseases on COVID-19 in China. Clin Res Cardiol. 2020;109:531-8.

17. Denova-Gutiérrez E, Lopez-Gatell H, Alomia-Zegarra JL, et al. The association between obesity, type 2 diabetes, and hypertension with severe COVID-19 on admission among Mexicans. Obesity. 2020. https://doi.org/10.1002/oby.22946.

18. Kronsteiner B, Chaichana P, Sumonwiriya M, Jenjaroen K, Chowdhury FR, Chumseng S, et al. Diabetes alters immune 
response patterns to acute melioidosis in humans. Eur J Immunol. 2019;49:1092-106.

19. Ayelign B, Negash M, Genetu M, Wondmagegn T, Shibabaw T. Immunological impacts of diabetes on the susceptibility of Mycobacterium tuberculosis. J Immunol Res. 2019;2019:1-8. https://doi.org/10.1155/2019/6196532.

20. Allard R, Leclerc P, Tremblay C, Tannenbaum TN. Diabetes and the severity of pandemic influenza a (H1N1) infection. Diabetes Care. 2010;33:1491-3.

21. Hernández JL, Buckley CJ. Mucormycosis. 2020 Jun 26. In: StatPearls [Internet]. Treasure Island (FL): StatPearls Publishing; 2020 Jan-. PMID: 31335084.

22. Roden MM, Zaoutis TE, Buchanan WL, Knudsen TA, Sarkisova TA, Schaufele RL, et al. Epidemiology and outcome of zygomycosis: a review of 929 reported cases. Clin Infect Dis. 2005:41:634-53.

23. Yousfi H, Ranque S, Rolain JM, Bittar F. In vitro polymyxin activity against clinical multidrug-resistant fungi. Antimicrob Resist Infect Control. 2019;8:66

24.• Soare AY, Watkins TN, Bruno VM. Understanding Mucormycoses in the age of "omics". Front Genet. 2020;11. https://doi.org/10. $3389 /$ fgene.2020.00699. In this review article it is discussed the specific contributions that NGS-based approaches have made to the field of mucormycosis including genome structure, drug resistance, diagnostic development, and fungus-host interactions.

25. Walther G, Wagner L, Kurzai O. Updates on the taxonomy of mucorales with an emphasis on clinically important taxa. J Fungi (Basel). 2019;5. https://doi.org/10.3390/jof5040106.

26. Gomes MZR, Lewis RE, Kontoyiannis DP. Mucormycosis caused by unusual mucormycetes, non-Rhizopus, -Mucor, and Lichtheimia species. Clin Microbiol Rev. 2011;24:411-45.

27. Hoffmann K, Pawłowska J, Walther G, Wrzosek M, de Hoog GS, Benny GL, et al. The family structure of the Mucorales: a synoptic revision based on comprehensive multigene-genealogies. Persoonia. 2013;30:57-76.

28. Dolatabadi S, de Hoog GS, Meis JF, Walther G. Species boundaries and nomenclature of Rhizopus arrhizus (syn. R. oryzae ). Mycoses. 2014:57:108-27.

29. Gryganskyi AP, Golan J, Dolatabadi S, et al. Phylogenetic and phylogenomic definition of Rhizopus species. G3 (Bethesda). 2018;8:2007-18.

30. Gryganskyi AP, Lee SC, Litvintseva AP, et al. Structure, function, and phylogeny of the mating locus in the Rhizopus oryzae complex. PLoS One. 2010;5. https://doi.org/10.1371/journal.pone.0015273.

31. Liu C, Feng X, Li Q, et al. Adiponectin, TNF- $\alpha$ and inflammatory cytokines and risk of type 2 diabetes: A systematic review and meta-analysis. Cytokine. 2016;86:100-9. This systematic review of prospective studies reports an association of elevated CRP, TNF- $\alpha$ and IL-6 levels with T2DM risk, concluding that these biomarkers are predictors of T2DM subjects.

32. Pavlou S, Lindsay J, Ingram R, et al. Sustained high glucose exposure sensitizes macrophage responses to cytokine stimuli but reduces their phagocytic activity. BMC Immunol. 2018;19:1-13.

33. Davies LC, Rice CM, McVicar DW, et al. Diversity and environmental adaptation of phagocytic cell metabolism. J Leukoc Biol. 2019;105:37-48.

34. Lecube A, Pachón G, Petriz J, et al. Phagocytic activity is impaired in type 2 diabetes mellitus and increases after metabolic improvement. PLoS One. 2011;6:6-11.

35. Toniolo A, Cassani G, Puggioni A, Rossi A, Colombo A, Onodera $\mathrm{T}$, et al. The diabetes pandemic and associated infections: suggestions for clinical microbiology. Rev Med Microbiol. 2019;30:1-17.

36. Spellberg B, Walsh TJ, Kontoyiannis DP, Edwards, Jr. J, Ibrahim AS. Recent advances in the management of mucormycosis: from bench to bedside. Clin Infect Dis. 2009;48:1743-51.
37. Ibrahim AS, Spellberg B, Walsh TJ, Kontoyiannis DP. Pathogenesis of mucormycosis. Clin Infect Dis. 2012;54:S16-22.

38. Lax C, Pérez-Arques C, Navarro-Mendoza MI, Cánovas-Márquez JT, Tahiri G, Pérez-Ruiz JA, et al. Genes, Pathways, and Mechanisms Involved in the Virulence of Mucorales. Genes 2020;11:317.

39. Reid G, Lynch JP 3rd, Fishbein MC, Clark NM. Mucormycosis. Semin Respir Crit Care Med. 2020;41(1):99-114. https://doi.org 10.1055/s-0039-3401992.

40. Farmakiotis D, Kontoyiannis DP. Mucormycoses. Infect Dis Clin N Am. 2016;30:143-63.

41. Ibrahim AS, Spellberg B, Walsh TJ, et al. Pathogenesis of mucormycosis. Clin Infect Dis. 2012;54:1-7.

42. Lax C, Pérez-arques C, Navarro-mendoza MI, et al. Genes, pathways, and mechanisms involved in the virulence of mucorales. Genes (Basel). 2020;11. https://doi.org/10.3390/genes11030317.

43. Petrikkos G, Tsioutis C. Recent advances in the pathogenesis of mucormycoses. Clin Ther. 2018;40:894-902.

44. Hassan MIA, Voigt K. Pathogenicity patterns of mucormycosis: epidemiology, interaction with immune cells and virulence factors. Med Mycol. 2019;57:S245-56.

45. Navarro-Mendoza MI, Pérez-Arques C, Murcia L, et al Components of a new gene family of ferroxidases involved in virulence are functionally specialized in fungal dimorphism. Sci Rep. 2018;8:1-13. https://doi.org/10.1038/s41598-018-26051-x.

46. Ibrahim AS, Spellberg B, Edwards J Jr. Iron acquisition: a novel perspective on mucormycosis pathogenesis and treatment. Curr Opin Infect Dis. 2008 Dec;21(6):620-5. https://doi.org/10.1097/ QCO.0b013e3283165fd1.

47. Andrianaki AM, Kyrmizi I, Thanopoulou K, et al. Iron restriction inside macrophages regulates pulmonary host defense against Rhizopus species. Nat Commun. 2018;9. https://doi.org/10.1038/ s41467-018-05820-2.

48. Agoro R, Taleb M, Quesniaux VFJ, et al. Cell iron status influences macrophage polarization. PLoS One. 2018;13. https://doi.org/10. 1371/journal.pone.0196921.

49. Schulze B, Rambach G, Schwartze VU, Voigt K, Schubert K, Speth $\mathrm{C}$, et al. Ketoacidosis alone does not predispose to mucormycosis by Lichtheimia in a murine pulmonary infection model. Virulence. 2017:8:1657-67.

50. Salazar-Tamayo G, López-Jácome LE, Resendiz-Sanchez J, Franco-Cendejas R, Rodriguez-Zulueta P, Corzo-León DE. Higher in vitro proliferation rate of Rhizopus oryzae in blood of diabetic individuals in chronic glycaemic control compared with non-diabetic individuals. Mycopathologia. 2017;182:1005-14.

51. Magdaleno F, Blajszczak C, Charles-Niño C, et al. Aminoguanidine reduces diabetes-associated cardiac fibrosis. Exp Ther Med. 2019;18. https://doi.org/10.3892/etm.2019.7921.

52. de Melo LGP, Nunes SOV, Anderson G, Vargas HO, Barbosa DS, Galecki P, et al. Shared metabolic and immune-inflammatory, oxidative and nitrosative stress pathways in the metabolic syndrome and mood disorders. Prog Neuro Psychopharmacol Biol Psychiatry. 2017:78:34-50.

53. Shiu RPC, Pouyssegur J, Pastan I. Glucose depletion accounts for the induction of two transformation-sensitive membrane proteins in Rous sarcoma virus-transformed chick embryo fibroblasts. Proc Natl Acad Sci U S A. 1977;74:3840-4.

54. Ting J, Lee AS. Human gene encoding the 78,000-dalton glucoseregulated protein and its pseudogene: structure, conservation, and regulation. DNA. 1988;7:275-86.

55. Hendershot LM, Valentine VA, Lee AS, Morris SW, Shapiro DN. Localization of the gene encoding human bip/grp78, the endoplasmic reticulum cognate of the hsp70 family, to chromosome $9 \mathrm{q} 34$. Genomics. 1994:20:281-4. 
56. Yang J, Nune M, Zong Y, Zhou L, Liu Q. Close and allosteric opening of the polypeptide-binding site in a human Hsp70 chaperone BiP. Structure. 2015;23:2191-203.

57. Roller C, Maddalo D. The molecular chaperone GRP78/BiP in the development of chemoresistance: mechanism and possible treatment. Front Pharmacol. 2013;4. https://doi.org/10.3389/fphar. 2013.00010.

58. Kwon JW, Jung I, Jee D. Glucose-regulated protein 78 in the aqueous humor in diabetic macular edema patients. Medicine (Baltimore). 2018;97. https://doi.org/10.1097/MD. 0000000000012757.

59. Ni M, Zhang Y, Lee AS. Beyond the endoplasmic reticulum: atypical GRP78 in cell viability, signalling and therapeutic targeting. Biochem J. 2011;434:181-8.

60. Ni M, Zhou H, Wey S, et al. Regulation of PERK signaling and leukemic cell survival by a novel cytosolic isoform of the UPR regulator GRP78/BiP. PLoS One. 2009;4. https://doi.org/10.1371/ journal.pone.0006868.

61. Sun FC, Wei S, Li CW, Chang YS, Chao CC, Lai YK. Localization of GRP78 to mitochondria under the unfolded protein response. Biochem J. 2006;396:31-9.

62. Gonzalez-Gronow M, Selim MA, Papalas J, et al. GRP78: a multifunctional receptor on the cell surface. Antioxid Redox Signal. 2009;11:2299-306.

63. Berger CL, Dong Z, Hanlon D, Bisaccia E, Edelson RL. A lymphocyte cell surface heat shock protein homologous to the endoplasmic reticulum chaperone, immunoglobulin heavy chain binding protein BIP. Int J Cancer. 1997;71:1077-85.

64. Crane ED, Al-Hashimi AA, Chen J, et al. Anti-GRP78 autoantibodies induce endothelial cell activation and accelerate the development of atherosclerotic lesions. JCI insight. 2018;3. https://doi. org/10.1172/jci.insight.99363.

65. Van Krieken R, Mehta N, Wang T, et al. Cell surface expression of 78-kDa glucose-regulated protein (GRP78) mediates diabetic nephropathy. J Biol Chem. 2019;294:7755-68.

66.• Liu M, Spellberg B, Phan QT, et al. The endothelial cell receptor GRP78 is required for mucormycosis pathogenesis in diabetic mice. J Clin Invest. 2010;120:1914-24. This study identified GRP78 receptor as a novel host endotelial cell receptor in mice that mediates invasion and damage of human endothelial cells by Rhizopus oryzae, mainly in uncontrolled T2DM subjects.

67.• Gebremariam T, Liu M, Luo G, et al. CotH3 mediates fungal invasion of host cells during mucormycosis. J Clin Invest. 2014;124: 237-50. This study elucidated that the presence of CotH in Mucorales can explain the specific susceptibility of DKA patients, who have increased GRP78 levels, to mucormycosis, identifying CotH as a promising therapeutic target for this affection.

68. Ha DP, Van Krieken R, Carlos AJ, et al. The stress-inducible molecular chaperone GRP78 as potential therapeutic target for coronavirus infection. J Inf Secur. 2020;81:452-82. https://doi.org/10. 1016/j.jinf.2020.06.017.

69.• Alqarihi A, Gebremariam T, Gu Y, et al. GRP78 and integrins play different roles in host cell invasion during mucormycosis. MBio. 2020;11. https://doi.org/10.1128/mBio.01087-20. In this article it is shown that Rhizopus delemar interacts with GRP78 on nasal epithelial cells via its spore coat protein CotH3 to invade and damage the nasal epithelial cells.
70. Shumilov E, Bacher U, Perske C, et al. In situ validation of the endothelial cell receptor GRP78 in a case of rhinocerebral mucormycosis. Antimicrob Agents Chemother. 2018;62. https:// doi.org/10.1128/AAC.00172-18.

71. Isticato R, Sirec T, Giglio R, et al. Flexibility of the prograamme of spore coat formation in Bacillus subtilis: bypass of CotE requirement by over-production of CotH. PLoS One. 2013;8. https://doi. org/10.1371/journal.pone.0074949.

72. Nguyen KB, Sreelatha A, Durrant ES, Lopez-Garrido J, Muszewska A, Dudkiewicz M, et al. Phosphorylation of spore coat proteins by a family of atypical protein kinases. PNAS. 2016;113: E3482-91.

73. Ibrahim AS, Kontoyiannis DP. Update on mucormycosis pathogenesis. Curr Opin Infect Dis. 2013;26:508-15.

74. Baldin C, Ibrahim AS. Molecular mechanisms of mucormycosisThe bitter and the sweet. PLoS Pathog. 2017;13. https://doi.org/10. 1371/journal.ppat.1006408.

75. Bouchara J, Oumeziane N, Lissitzky J, Larcher G, Tronchin G, Chabasse D. Attachment of spores of the human pathogenic fungus Rhizopus oryzae to extracellular matrix components - PubMed. Eur J Cell Biol. 1996;70:76-83.

76. Oku S, Takeshita T, Futatsuki T, et al. Disrupted tongue microbiota and detection of nonindigenous bacteria on the day of allogeneic hematopoietic stem cell transplantation. PLoS Pathog. 2020;16:118.

77. Salmanton-García J, Seidel D, Koehler P, Mellinghoff SC, Herbrecht R, Klimko N, et al. Matched-paired analysis of patients treated for invasive mucormycosis: standard treatment versus posaconazole new formulations (MoveOn). J Antimicrob Chemother. 2019;74:3315-27.

78. Macedo D, Leonardelli F, Dudiuk C, et al. In vitro and in vivo evaluation of voriconazole-containing antifungal combinations against mucorales using a Galleria mellonella model of mucormycosis. J Fungi. 2019;5. https://doi.org/10.3390/ jof5010005.

79. Tominaga T, Uchida R, Koyama N, Tomoda H. Anti-Rhizopus activity of tanzawaic acids produced by the hot spring-derived fungus Penicillium sp. BF-0005. J Antibiot (Tokyo). 2018;71:626-32.

80. Carter-House, D., Chung, J., McDonald, S., Mauck, K. \& Stajich, J. E. Volatiles from Serratia marcescens, S. proteamaculans, and Bacillus subtilis Inhibit Growth of Rhizopus stolonifer and Other Fungi. bioRxiv 2020. 2020.09.07.286443.

81. Sephton-Clark PCS, Muñoz JF, Ballou ER, et al. Pathways of pathogenicity: transcriptional stages of germination in the fatal fungal pathogen Rhizopus delemar. mSphere. 2018;3. https://doi.org/10. $1128 / \mathrm{msphere} .00403-18$. This study provides a greater understanding of the regulation of germination and highlights processes involved in transforming Rhizopus delemar from a single-cellular to multicellular organism.

82. Malavia D, Gow NAR, Usher J. Advances in molecular tools and in vivo models for the study of human fungal pathogenesis. Microorganisms. 2020;8. https://doi.org/10.3390/ microorganisms 8060803 .

Publisher's Note Springer Nature remains neutral with regard to jurisdictional claims in published maps and institutional affiliations. 